\title{
Spatial Organization in Two-Species Annihilation
}

\author{
S. Redner $^{1}$ and F. Leyvraz ${ }^{2}$
}

\begin{abstract}
The spatial structure of reactants in the two-species annihilation reaction $A+B \rightarrow 0$ is described. In one dimension, we investigate the distribution of domain sizes and the distributions of nearest-neighbor distances between particles of the same and of opposite species. The latter two quantities are characterized by a new length scale which is intermediate to the domain size $t^{1 / 2}$ and the typical interparticle spacing $t^{1 / 4}$. A scaling argument suggests that the typical distance between particles of opposite species, or equivalently the gaps between domains, grows as $t^{\zeta}$, with $\zeta=3 / 8$ and $1 / 3$, respectively, in spatial dimension $d=1$ and 2 . The average density profile of a single domain is spatially nonuniform, with the density decaying to zero linearly as the domain edge is approached. This behavior permits a determination of the distribution of nearest-neighbor distances of same-species reactants. The corresponding moments of this distribution exhibit multiscaling which involves geometric averages of different powers of the domain size and the interparticle spacing.
\end{abstract}

KEY WORDS: Spatial organization; domain profile; interparticle distances.

\section{INTRODUCTION}

In the irreversible diffusion-limited two-species annihilation reaction $\mathrm{A}+\mathrm{B} \rightarrow 0$, it is widely appreciated that the rate equation does not account for the kinetics of the reaction process. For an initially random distribution of A's and B's at equal concentrations, the density decays as $t^{-d / 4}$ when the spatial dimension $d$ is less than four, ${ }^{(1-5)}$ compared to the mean-field decay of $t^{-1}$. This slower decay can be viewed as stemming from an effective "repulsion" between the A and B species. Whenever an A and a B are within the reaction range, the pair is annihilated. Thus the reaction process

\footnotetext{
${ }^{1}$ Center for Polymer Studies and Department of Physics, Boston University, Boston, Massachusetts 02215.

${ }^{2}$ Instituto de Fisica, Laboratorio de Cuernavaca, UNAM, Mexico.
} 
itself provides a mechanism which attempts to keep A's and B's separated, with a corresponding reduction in the reaction rate. For a Poissonian initial distribution of reactants, this separation mechanism causes the initial density fluctuations to become more pronounced, and the system organizes into continuously growing single-species domains.

These features of the reaction kinetics and the spatial distribution of reactants can be understood by a simple qualitative approach which is based on following the temporal evolution of the initial density fluctuations. Consider a spatial region of linear dimension $l$ and corresponding volume $l^{d}$. In an initial state with equal densities of the two species, the numbers of A's and B's are, respectively

$$
\begin{aligned}
& N_{A}(0)=c(0) l^{d} \pm\left[c(0) l^{d}\right]^{1 / 2} \\
& N_{B}(0)=c(0) l^{d} \pm\left[c(0) l^{d}\right]^{1 / 2}
\end{aligned}
$$

Here $c(0)$ denotes the initial concentration of each species, and the \pm terms represent typical fluctuations in particle number in a volume $l^{d}$. After a time $t \sim l^{2} / D$, there will be essentially complete diffusive mixing of particles within the volume. Consequently the "extensive" parts of the particle numbers cancel in pairs, leaving behind only the residual fluctuations. Thus the remaining number of particles (of the local majority species) is of order $N_{>} \sim\left[c(0) l^{d}\right]^{1 / 2}$ and the local concentration is $c_{>} \sim N_{>} / l^{d}$. In this argument, the contributions due to inflow and outflow of particles from the volume are ignored. Since this fluctuation-induced behavior occurs in every domain the average concentration equals this local concentration. Therefore

$$
c(t)=c_{>} \propto \frac{\left[c(0) l^{d}\right]^{1 / 2}}{l^{d}} \propto t^{-d / 4}
$$

Accordingly, an initially homogeneous system coarsens into alternating A-rich and B-rich domains ${ }^{(1-9)}$ of linear dimension proportional to $t^{1 / 2}$. Since the density decays as $t^{-d / 4}$, the typical interparticle spacing grows as $c(t)^{-1 / 4} \sim t^{1 / 4}$. Thus, at least two length scales are necessary to account for spatial distributions of particles. One of our main results is that yet another length scale, which is intermediate to the interparticle spacing and the domain size, is needed to account for the spatial distribution of reactants, ${ }^{(10)}$ when $1 \leqslant d \leqslant 2$. We also find interesting spatial structure for the "reaction zone," ${ }^{(1-13)}$ i.e., the interfacial region between A and $\mathrm{B}$ domains where the reaction actually takes place. Correspondingly, the density profile within a single domain is spatially nonuniform, with the particle density decaying to zero as the domain boundary is approached. Conse- 
quently, the distribution of distances ${ }^{3}$ between nearest-neighbor samespecies particles is determined by a competition between the relatively closely-spaced particles in the domain core and the more widely-separated particles in the domain periphery. This feature has interesting ramifications for the moments of the distances between same-species particles.

\section{SPATIAL DISTRIBUTION OF REACTANTS IN ONE DIMENSION}

To describe the distribution of particles in one dimension, it is useful to consider the following three distribution functions (Fig. 1). (a) The probability that a domain has a length equal to $x$ at time $t, P_{\text {dom }}(x, t)$, and the probabilities that the nearest-neighbor distance between particles of (b) the same and (c) the opposite species equals $x, P_{\mathrm{AA}}(x, t)$ and $P_{\mathrm{AB}}(x, t)$, respectively. The latter distance is the same as the length of the gaps. between domains. We have performed numerical simulations to measure these quantities which are based on averaging 80 configurations of up to 11,222 time steps on a chain of 500,000 sites at an initial density of 0.4 for each species.

The average domain size increases as $t^{1 / 2}$, and the distribution of domain sizes is fairly sharply peaked about this average value (Fig. 2). Visually, the distribution of domain sizes is well fit by the form $P_{\mathrm{dom}}(x, t) \sim A x e^{-x / x_{\mathrm{dom}}(t)}$, with $x_{\mathrm{dom}}(t)$ increasing as $t^{1 / 2}$. This functional form also accords reasonably well with the temporal behavior of the positive integer moments of the distribution. The domain boundaries evolve essentially as annihilating one-dimensional random walks, a system for which much is already known about the time dependence of the density (e.g., refs. 15). The distribution of domain lengths is equivalent to the distance distribution of nearest neighbors in the annihilation process. Apparently, there are no rigorous results for the behavior of this latter distribution. ${ }^{(16)}$ Figure 3 shows the time evolution of the domain size distribution. The number of domains of a fixed length $2 L, n_{2 L}(t)$, initially grows

\footnotetext{
${ }^{3}$ Various related aspects of interparticle distribution functions in two-species annihilation
} were obtained in ref. 14.

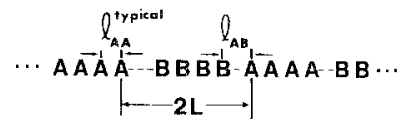

Fig. 1. Definition of the typical distance between neighboring particles of the same species $l_{\mathrm{AA}}^{\text {typal }}$, the gap length (or nearest neighbor distance between unlike species) $l_{\mathrm{AB}}$, and the domain diameter $2 L$. 

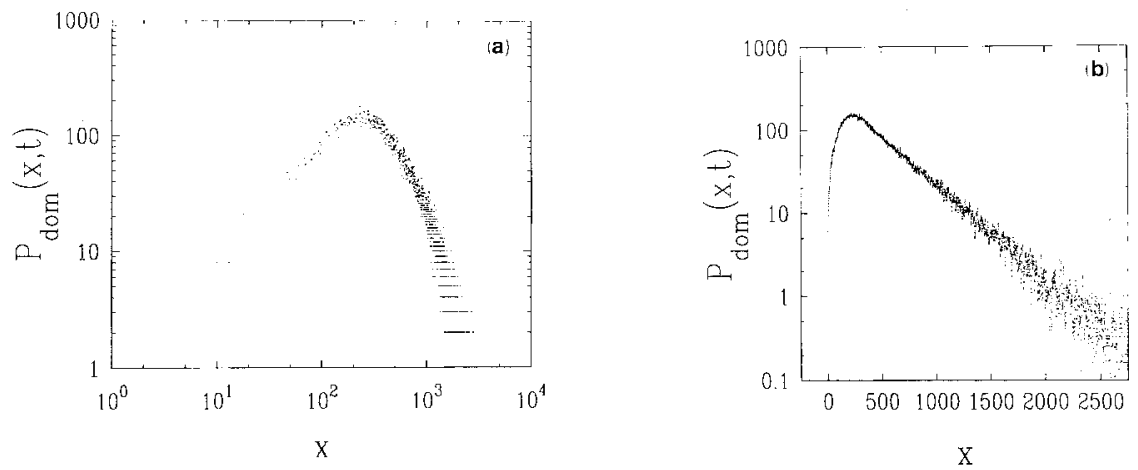

Fig. 2. The distribution of domain sizes $P_{\mathrm{dom}}(x, t)$ at $t=11,222$ on (a) a double-logarithmic scale to exhibit the linear behavior at small distances, and (b) a semilogarithmic scale to exhibit the large-distance exponential tail. In the latter case, the data have been smoothed by performing an average of the distribution over 21 consecutive points.

with time until the typical domain size reaches $2 L$. At longer times, $n_{2 L}(t)$ appears to decay as $t^{-3 / 2}$.

More interesting behavior occurs in the distribution of interdomain gap lengths. For the average size of the gaps, one naively expects that the effective repulsion between unlike species would lead to a gap size which is larger than the average distance between same-species pairs. From our simulations, the gap size appears to increase as $t^{\zeta}$, with $\zeta \approx 3 / 8$ (Fig. 4). A rough derivation of this putative new exponent will be given in the next section. Furthermore, the distribution of gap sizes also appears to be well fit by the form $P_{\mathrm{AB}}(x, t) \sim A x e^{-x / x_{\mathrm{AB}}}$ with $x_{\mathrm{AB}} \propto t^{3 / 8}$ (Fig. 5).

There is a qualitatively different form for the distribution of distances between particles of the same species. As a function of $x, P_{\mathrm{AA}}(x, t)$ decays

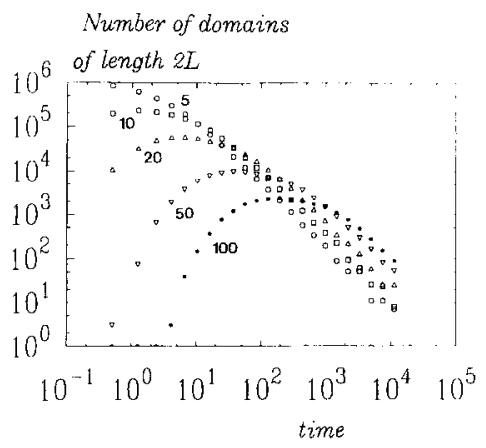

Fig. 3. Time evolution of the distribution of domain lengths. 


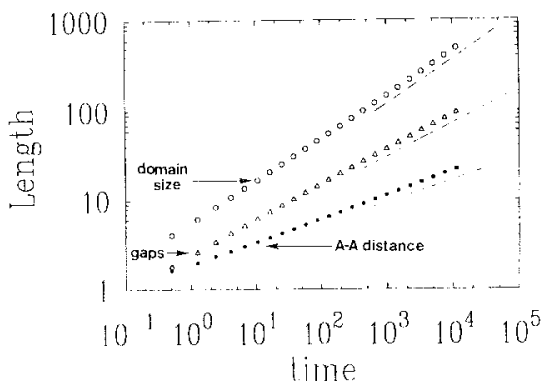

Fig. 4. Time dependence of the average spacing between particles of the same species, the average gap size, and the average domain size. The dashed lines of respective slopes $1 / 4,3 / 8$, and $1 / 2$ are guides to the eye.

monotonically, with an apparently simple exponential tail at large distances, characteristic of a random distribution of particles (Fig. 6). We shall argue below that this form for $P_{\mathrm{AA}}(x, t)$ can be derived by first assuming that there is a local Poisson distance distribution for particles of the same species, in which the average spacing depends on the relative position of the neighboring particles within the domain. Particles are more closely spaced in the domain core, and are progressively more widely separated as the domain boundary is approached. By averaging the local Poisson distributions over this domain density profile, a form for $P_{\mathrm{AA}}(x, t)$ is obtained which is in reasonable agreement with the simulation data.
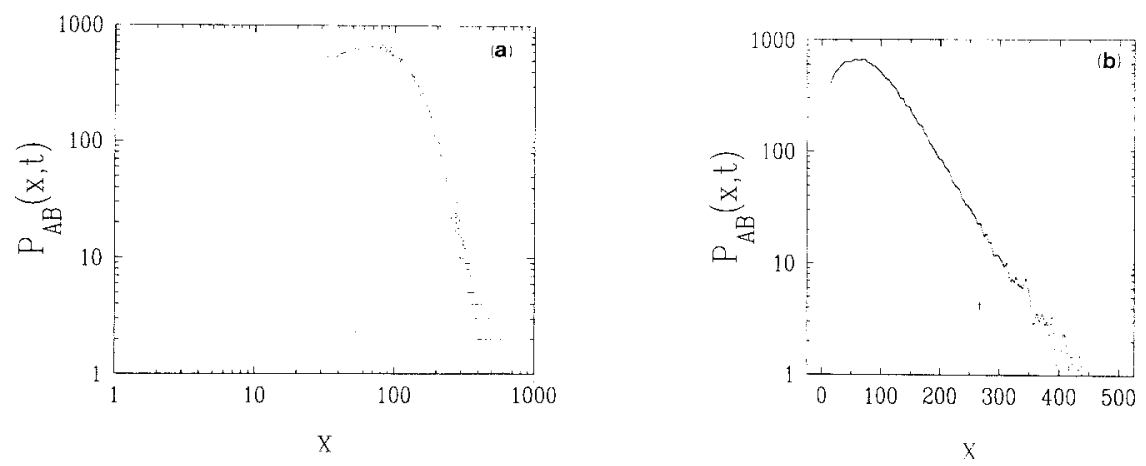

Fig. 5. The distribution of gap lengths between domains $P_{\text {dom }}(x, t)$ at $t=11,222$ on (a) a double-logarithmic scale to exhibit the linear behavior at small distances, and (b) a semilogarithmic scale to exhibit the large-distance exponential tail. The data are smoothed by averaging the distribution over 11 consecutive points. 


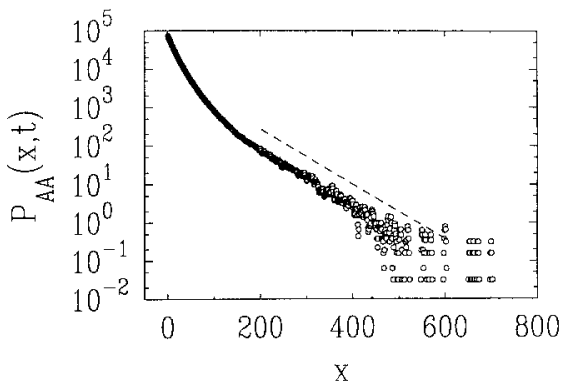

Fig. 6. The distribution of the distance between nearest neighbors of the same species $P_{\text {AA }}(x, t)$ at $t=11,222$ on a semilogarithmic scale to exhibit the large-distance exponential tail. The data are smoothed by averaging the distribution over 5 consecutive points. The slope of the best-fit straight line that fits the asymptotic decay (dashed and offset) vanishes as $t^{-3 / 8}$.

\section{THE AVERAGE PROFILE OF A SINGLE DOMAIN}

The striking new result from our simulations is that the average distance between domains is an apparent new length scale, independent of the interparticle spacing or the domain size. The time dependence of this new length can be appreciated from the following qualitative argument. ${ }^{(10)}$ Let us postulate that the gaps between domains are of (unknown) length $l_{\mathrm{AB}} \sim t^{\zeta}$. We now give an alternative derivation for the known one-dimensional decay of the density in terms of $l_{\mathrm{AB}}$, from which the time dependence of the gap length can be inferred. In a time interval $\Delta t \propto l_{\mathrm{AB}}^{2}$, there will typically be one reaction in each gap. Since two particles per domain are annihilated by these reactions, the concentration change $\Delta c$ is proportional to the inverse domain size $t^{-1 / 2}$. Hence

$$
\frac{\Delta c}{\Delta t} \approx \frac{d c}{d t} \propto t^{-1 / 2-2 \zeta}
$$

Thus, in order for $c(t)$ to be proportional to $t^{-1 / 4}$, it is necessary that $\zeta=3 / 8$. This result is in excellent agreement with the numerical results quoted above.

To gain a deeper appreciation for the role of this new length scale, it is useful to examine the average density profile of a single domain (Fig. 7). For this purpose, we first define a local spatial coordinate with respect to the center of each domain. The boundaries of the domain are defined to be the first particle of the opposite species on either side of the domain. These two bounding particles impose absorbing boundary conditions for the particles within the domain. In order that the average profile over all domains has a well-defined boundary condition, we rescale each domain so that they 

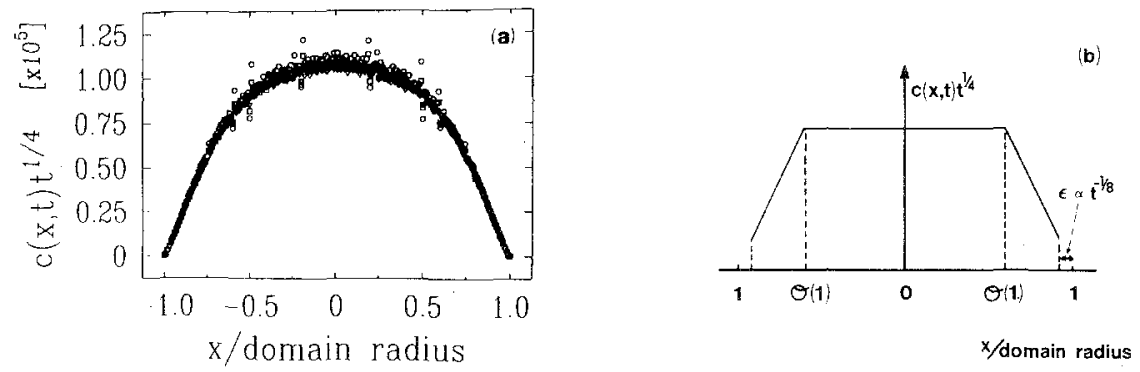

Fig. 7. (a) The scaled domain profile for $t=194(\bigcirc), t=1477(\square)$, and $t=11,222(\nabla)$. The local coordinate is defined as the distance from the center of the domain. The scaled profile suggests the trapezoidal profile (b).

all have the same length before superposing the individual density profiles. This procedure is validated, to some degree, by the relative sharpness of the domain size distribution. In this rescaling, particles are placed at the closest integer point to the rescaled position. This prescription introduces some truncation errors at early times, but is a negligible effect at large times.

The resulting density profiles can now be scaled by multiplying each profile at time $t$ by $t^{1 / 4}$ to compensate for the overall $t^{-1 / 4}$ decay of the density. As a function of the scaled abscissa, the scaled profile resembles onehalf of a cosine wave, but with a somewhat flattened central peak (Fig. 7). The quality of the scaling is good, except that there is a very small but systematic decrease in the scaled profiles at different times. The best scaling is actually achieved by multiplying the profiles at different times by $t^{z}$ with $z \approx 0.265$. Furthermore, domains containing just a single particle give rise to an anomalous peak at $x=0$ whose value is more than a factor of two larger than the scaling profile. Except for these caveats, the density profiles scale nicely.

The cosinusoidal shape of the density profile can be understood in terms of a crude model in which the particles inside an A domain (for example) are diffusing independently within the region defined by the two enclosing $\mathrm{B}$ domains whose edges are systematically receding at a rate proportional to $t^{1 / 2}$. This is meant to mimic the stochastic diffusive motion of the domain boundary. In a continuum approach, the concentration of A's thus obeys the diffusion equation

$$
\frac{\partial c}{\partial t}=\frac{\partial^{2} c}{\partial x^{2}}
$$

subject to the boundary conditions $c( \pm L(t), t)=0$, with the domain radius $L(t)$ increasing as $t^{1 / 2}$. A growth of the radius which is of order $t^{1 / 2}$ or less 
is sufficiently slow that one can apply the adiabatic approximation (e.g., ref. 17). In this approach, the spatial variation of the density profile is the same as a system with static absorbing boundaries, and only the overall temporal decay of the probability within the domain is affected by the domain boundary motion. The adiabatic approximation gives, for the local density in a domain,

$$
\begin{aligned}
c(x, t) & =\cos [\pi x / 2 L(t)] \exp \left[- \text { const } \times \int_{0}^{t} d t^{\prime} / L\left(t^{\prime}\right)^{2}\right] \\
& =\cos [\pi x / 2 L(t)] t^{- \text {const }}
\end{aligned}
$$

This approximation cannot be refined to the degree necessary to find the exponent that characterizes the decay of the density, however, owing to the crudeness of the single-domain model.

There are several noteworthy aspects of the density profile. First, the density decays linearly to zero as the domain boundary is approached (Fig. 7). Second, the density profile can be roughly divided into a core region, where the local density is slowly varying, and an interfacial layer, with each region comprising a finite fraction of the domain length. In the domain core, the local density decays as $t^{-1 / 4}$, corresponding to the typical interparticle spacing growing as $t^{1 / 4}$. As the domain boundary is approached, the density decreases monotonically and the typical interparticle distance increases accordingly. Very roughly, these essential features of the scaled domain profile can be accounted for by the following trapezoidal form (Fig. 7b):

$$
\rho(z) \equiv c(x, t) t^{1 / 4}= \begin{cases}\rho_{0}, & |z| \leqslant z^{*} \\ \rho_{0}(1-|z|), & z^{*}<|z|<1-\varepsilon\end{cases}
$$

Here $\rho_{0}$ and $z^{*}$ are constants, with $z^{*}$ less than, but of order unity, and $z$ is the scaled spatial coordinate, defined by $z=x / L(t)$. The upper limit for $|z|$ on the second line of Eq. (6) reflects the fact that there are no particles within a scaled distance of $\varepsilon \equiv l_{\mathrm{AB}} / L(t) \sim t^{-1 / 8}$ from the domain edge. While the trapezoidal form is not correct in detail, it provides a useful idealization from which many of the interesting features of the spatial distribution of reactants can be obtained with minimal calculational effort.

It is also worth mentioning that the time dependence of the typical gap size $l_{\mathrm{AB}}$ can be obtained directly from the trapezoidal density profile. ${ }^{(18,19)}$ Consider a shifted local coordinate $y=L+x$ whose origin is at the left edge of the domain. Then the position of the leftmost particle in the domain is defined by the condition

$$
\int_{0}^{l_{A B}} c(y, t) d y=1
$$


This merely states that of the order of one particle is within the interdomain gap. According to the trapezoidal profile, $c(y, t) \propto y f(t)$ for $y<t^{1 / 2}$ and $c(y, t)$ approaches a constant limiting value proportional to $t^{-1 / 4}$ when $y \approx t^{1 / 2}$. Matching these two limiting behaviors leads to $f(t) \propto t^{-3 / 4}$. Using this result for $c(y, t)$ in Eq. (7) immediately gives $l_{\mathrm{AB}} \sim t^{3 / 8}$.

In addition to finding the typical value of $l_{\mathrm{AB}}$, this extreme value approach can be easily extended to find the probability distribution for $l_{\mathrm{AB}}$, namely $P_{\mathrm{AB}}(x, t)$. Following the approach of ref. 19 step by step, it is easy to determine that $P_{\mathrm{AB}}(x, t) \propto x e^{-\left(x / x_{\mathrm{AB}}\right)^{2}}$, in contrast to the simple exponential decay observed in the simulations. The discrepancy apparently stems from the fact that the analytical approach assumes a static absorbing point at the domain boundary, while in fact, the boundary point is actually undergoing diffusive motion.

\section{IMPLICATIONS OF THE INHOMOGENEOUS DOMAIN PROFILE}

The properties of the domain profile can be exploited to determine both the functional form of the distribution $P_{\mathrm{AA}}(x, t)$ and the time dependence of the corresponding reduced moments $M_{n} \equiv\left\langle l_{\mathrm{AA}}^{n}\right\rangle^{1 / n}$, defined by

$$
\left\langle l_{\mathrm{AA}}^{n}\right\rangle=\int_{0}^{\infty} x^{n} P_{\mathrm{AA}}(x, t) d x
$$

According to the trapezoidal form, the reduced moments are

$$
M_{n} \approx t^{1 / 4}\left(2 \int_{0}^{z^{*}} \frac{d z}{\rho_{0}^{n}}+2 \int_{z^{*}}^{1-\varepsilon} \frac{d z}{\rho_{0}^{n}(1-z)^{n}}\right)^{1 / n}
$$

In this expression, the $n$th moment of the nearest-neighbor distance between same-species particles is obtained by averaging the local nearestneighbor distance over the extent of the domain. This local nearestneighbor distance is simply $\rho(z)^{-1}$, with $\rho(z)=\rho_{0}$ in the domain core $\left(|z| \leqslant z^{*}\right)$, and with $\rho(z)=\rho_{0}(1-|z|)$ near the domain boundary. The interesting aspect of the above expression is that the dominant contribution to $M_{n}$ originates from different terms in Eq. (9) as $n$ is varied. For $n<1$, the integral is dominated by the first (constant) term in the parentheses. For $n=1$, however, the second term gives rise to a logarithmic singularity 
at the upper limit, while for $n>1$, the second term dominates the entire expression. The result of this straightforward calculation is

$$
M_{n} \sim \begin{cases}t^{1 / 4}, & n<1 \\ t^{1 / 4} \ln t, & n=1 \\ t^{(3 n-1) / 8 n}, & n>1\end{cases}
$$

Thus, there is a logarithmic factor in the ratio between the average and typical distances between nearest-neighbor particles of the same species, and a power-law diverging factor for the higher moments. For $n>1, M_{n}$ can be expressed in terms of the basic length scales in the system as

$$
M_{n} \sim l_{\mathrm{AA}}^{\text {typical }}\left(\frac{l_{\mathrm{dom}}}{l_{\mathrm{AB}}}\right)^{(n-1) / n}
$$

As $n \rightarrow \infty$, the reduced moment is dominated by the contribution from the sparsely populated region near the periphery of the domain where nearestneighbor particles are separated by a distance that grows as $t^{3 / 8}$.

These findings are corroborated by simulations (Fig. 8). For various $n>1$, double-logarithmic plots of $M_{n}$ versus $t$ yield straight lines of different slopes, with exponent values that are in good agreement with Eq. (11). For $n<1$, the $M_{n}$ appear to approach a common limit, asymptotically, as expected (Fig. 9). The slopes of the straight lines that pass through successive pairs of data points roughly extrapolate to exponent values of $0.26-0.27$. Finally, for $n=1$, the successive slopes are systematically increasing with time and extrapolate to an exponent value of about 0.29 . The gradual increase of the exponent does not accord with a logarithmic correction, although the value of the apparent exponent is in the range that is expected when a quantity which varies as $t^{1 / 4} \ln t$ is fit to a simple power law. We do not fully understand the source of these various small discrepancies with Eq. (11).

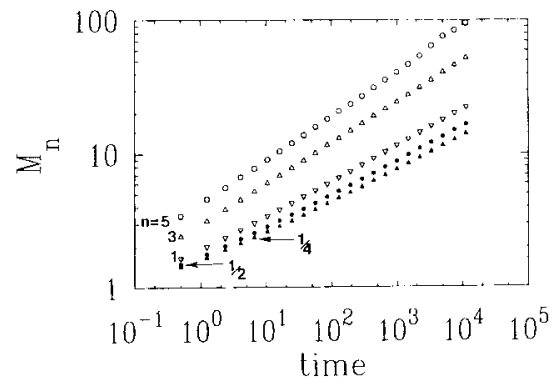

Fig. 8. Double-logarithmic plots of the reduced moments $M_{n}$ versus time. 

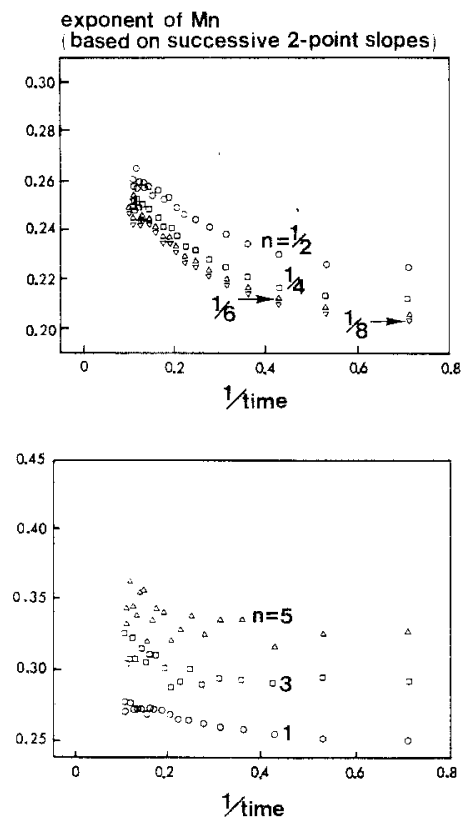

Fig. 9. The long-time behavior of the slopes between successive pairs of data points for $M_{n}$ to provide an estimate for the exponents that characterize their time dependence.

A second consequence of the spatially varying domain profile is that it provides a calculational framework with which we can give a rough derivation for the form of $P_{\mathrm{AA}}(x, t)$. Let us assume that particles are distributed approximately at random according to the local density $c(\xi, t)$, where $\xi$ is the local coordinate inside a single domain. Then the probability of finding a nearest neighbor distance between particles of the same species equal to $x$ at spatial location $\xi$ is

$$
P_{\mathrm{AA}}(\xi ; x, t)=c(\xi, t) e^{-x c(\xi, t)}
$$

The average probability of finding a spacing equal to $x$ is now obtained by averaging the local probability over a domain. In terms of the scaled local coordinate $z \propto \xi / t^{1 / 2}$ and the scaled density $\rho(z) \propto c(\xi, t) t^{1 / 4}$, this average becomes

$$
P_{\mathrm{AA}}(x, t) \propto t^{-1 / 4} \int_{0}^{1-\hat{\varepsilon}} d z \rho(z) e^{-x t^{-1 / 4} \rho(z)}
$$


This value of the integral is asymptotically dominated by the endpoint contribution associated with the upper limit on the integral. Thus for the asymptotic behavior of the integral, only the portion of the domain profile where there is a linear variation of density with position enters into the result. Consequently, we can use the trapezoidal domain profile in performing the integration in Eq. (13). In terms of the scaled spacing $\sigma \equiv s / t^{1 / 4}$, the result of performing the integral is

$$
P_{\mathrm{AA}}(\sigma) \propto F(\sigma) \exp \left(- \text { const } \times \sigma t^{-1 / 8}\right)
$$

with $F(\sigma) \propto \mathcal{O}\left(\sigma^{-2}\right)$ as $\sigma \rightarrow \infty$. From this form, it is also possible to obtain the moments $M_{n}$ already derived in Eq. (10). We expect to observe the power-law form for $F(\sigma)$ at a length scale intermediate to the typical and the largest interparticle spacing, a range which unfortunately grows only as $t^{1 / 8}$. Thus the largest time in our one-dimensional simulation, $t=11,222$, does not appear to be long enough to yield good numerical evidence for this power law; however, the data clearly exhibit the asymptotic exponential decay, with the correct time dependence of the characteristic decay length (Fig. 6).

\section{DOMAIN PROFILE IN TWO DIMENSIONS}

The earlier discussion about the interfacial region between domains can be readily extended to arbitrary spatial dimensions less than 2 . In analogy with the one-dimensional case, we hypothesize that a domain in

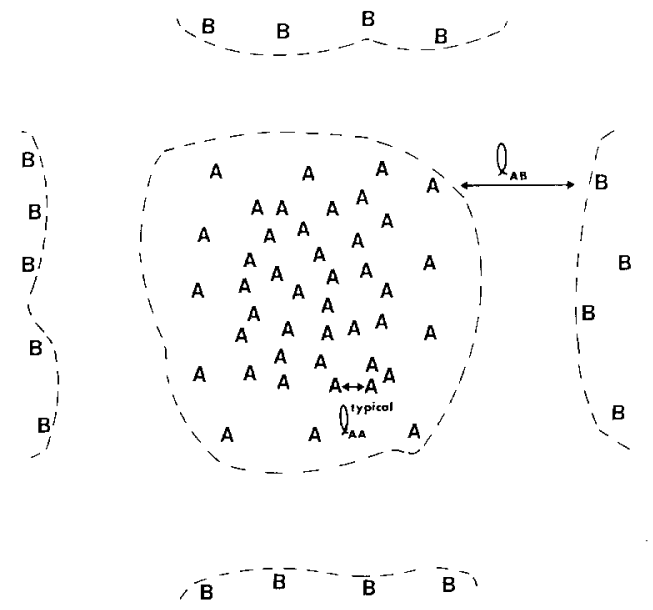

Fig. 10. Schematic illustration of a typical domain in two dimensions, showing the larger interparticle spacing in the interfacial region. 
spatial dimension $d<2$ has a core region of approximately constant density and a surrounding $(d-1)$-dimensional "skin" where the density vanishes as the domain edge is approached (Fig. 10).

To estimate the gap distance $l_{A B}$, we assume that the particles at the outer edge of the depletion zone are also separated by a distance which scales as $l_{\mathrm{AB}}$. This behavior is precisely what is observed in the one-dimensional case. Consequently, the number of boundary particles varies as $\left(t^{1 / 2} / l_{\mathrm{AB}}\right)^{d-1}$. Then in a time interval which is proportional to $l_{\mathrm{AB}}^{2}$, each of these boundary particles reacts, for $d \leqslant 2$, leading to a density change $\Delta c \sim\left(t^{1 / 2} / l_{\mathrm{AB}}\right)^{d-1} / t^{d / 2}$. In close analogy with Eq. (3), we now find

$$
\frac{d c}{d t} \sim t^{-1 / 2} l_{\mathrm{AB}}^{-(d+1)}
$$

Matching the resulting solution with the known decay of $c(t) \sim t^{-d / 4}$ for $d \leqslant 4$ yields $\zeta=(d+2) / 4(d+1)$. We expect that this dimension dependence for $\zeta$ holds only in the range $1 \leqslant d \leqslant 2$. For $d>2$, only a small fraction of the particles on the domain boundary react within the time interval $\Delta t \propto l_{\mathrm{AB}}^{2}$. Consequently, we expect that there is a much less pronounced depletion layer in this case. Simulations in two dimensions give a value for $\zeta$ which is close to the predicted value of $1 / 3$. These simulations also reveal an average density profile for a single domain which is qualitatively similar to the one-dimensional case, except that the depletion layer is confined to a much smaller fraction of the total extent of the domain.

\section{ACKNOWLEDGMENTS}

We thank D. ben-Avraham, A. Georges, and H. Park for helpful discussions. We gratefully acknowledge grants DAAL03-89-K-0025 from the Army Research Office and INT-8815438 from the National Science Foundation (S.R.), and a grant from CONACYT (F.L.) for partial support of this research.

\section{REFERENCES}

1. Ya. B. Zeldovich and A. A. Ovchinnikov, Chem. Phys. 28:215 (1978).

2. D. Toussaint and F. Wilczek, J. Chem. Phys. 78:2642 (1983).

3. K. Kang and S. Redner, Phys. Rev. Lett. 52:955 (1984); Phys. Rev. A 32:435 (1985).

4. G. Zumofen, A. Blumen, and J. Klafter, $J$. Chem. Phys. 82:3198 (1985).

5. M. Bramson and J. L. Lebowitz, Phys. Rev. Lett. 61:2397 (1988).

6. L. W. Anacker and R. Kopelman, Phys. Rev. Lett. 58:289 (1987); L. W. Anacker and R. Kopelman, J. Chem. Phys. 91:5555 (1987).

7. D. ben-Avraham and C. R. Doering, Phys. Rev. A 38:3035 (1988). 
8. K. Lindenberg, B. J. West, and R. Kopelman, Phys. Rev. Lett. 60:1777 (1988).

9. E. Clément, L. M. Sander, and R. Kopelman, Phys. Rev. A 39:6455 (1989).

10. F. Leyvraz and S. Redner, Phys. Rev. Lett. 66:2168 (1991).

11. L. Gálfi and Z. Rácz, Phys. Rev. A 38:3151 (1988).

12. Z. Jiang and C. Ebner, Phys. Rev. A 42:7483 (1990).

13. H. Taitlebaum, S. Havlin, J. E. Kiefer, B. Trus, and G. H. Weiss, preprint.

14. P. Argyrakis and R. Kopelman, Phys. Rev. A 41:2121 (1990).

15. M. Bramson and D. Griffeath, Z. Wahrsch. Verw. Gebiete 53:183 (1980); D. C. Torney and H. M. McConnell, Proc. R. Soc. Lond. A 387:147 (1983); A. A. Lushnikov, Sov. Phys. JETP 64:811 (1986); J. L. Spouge, Phys. Rev. Lett. 60:873 (1988).

16. C. R. Doering and D. ben-Avraham, Phys. Rev. A 38:3035 (1988); D. ben-Avraham, M. A. Burschka, and C. R. Doering, J. Stat. Phys. 60:695 (1990).

17. L. D. Landau and E. M. Lifshitz, Quantum Mechanics (Pergamon Press, New York, 1977).

18. G. H. Weiss, S. Havlin, and R. Kopelman, Phys. Rev. A 39:466 (1989); S. Havlin, H. Larralde, R. Kopelman, and G. H. Weiss, Physica $A$ 169:337 (1990).

19. S. Redner and D. ben-Avraham, J. Phys. A 23:L1169 (1990). 